\title{
Carvedilol Phosphate
}

National Cancer Institute

\section{Source}

National Cancer Institute. Carvedilol Phosphate. NCI Thesaurus. Code C65292.

The phosphate salt form of carvedilol, a racemic mixture and adrenergic blocking agent with antihypertensive activity and devoid of intrinsic sympathomimetic activity. The S enantiomer of carvedilol nonselectively binds to and blocks beta-adrenergic receptors, thereby exerting negative inotropic and chronotropic effects, and leading to a reduction in cardiac output. In addition, both enantiomers of carvedilol bind to and block alpha 1adrenergic receptors, thereby causing vasodilation and reducing peripheral vascular resistance. 